\title{
Diversity of Gut Methanogens and Functional Enzymes Associated With Methane Metabolism in Smallholder Dairy Cattle
}

Denis K. Ng'etich ( $\nabla$ ngetichkipkorirdenis@gmail.com )

University of Nairobi College of Agriculture and Veterinary Sciences https://orcid.org/0000-0002-13526445

Rawlynce C. Bett

University of Nairobi College of Agriculture and Veterinary Sciences

Charles K. Gachuiri

University of Nairobi College of Agriculture and Veterinary Sciences

Felix M. Kibegwa

University of Nairobi College of Agriculture and Veterinary Sciences

\section{Research Article}

Keywords: Ruminants, species, greenhouse, Enzymes, Kenya, Tanzania

Posted Date: January 3rd, 2022

DOI: https://doi.org/10.21203/rs.3.rs-1120235/v1

License: (9) This work is licensed under a Creative Commons Attribution 4.0 International License.

Read Full License 


\section{Abstract}

Methane is a greenhouse gas with disastrous consequences when released to intolerable levels. Ruminants produce methane during gut fermentation releasing it through belching and/or flatulence. To better understand the diversity of methanogens and functional enzymes associated with methane metabolism in dairy cows, 48 samples; six rumen and 42 dung contents were collected and analyzed using a shotgun metagenomic approach. The results indicated archaea from 5 phyla, 9 classes, 16 orders, 25 families, 59 genera, and 87 species. Gut sites significantly contributed to the presence and distribution of various methanogens $(P<0.01)$. The class Methanomicrobia was abundant in the rumen samples ( 39\%) and in dung ( 44\%). The most abundant ( 17\%) methanogen species identified was Methanocorpusculum labreanum. However, some taxonomic classes were not classified ( $6 \%$ in the rumen and $\sim 4 \%$ in the dung). Furthermore, five functional enzymes: Glycine/Serine hydroxy methyltransferase, Formylmethanofuran-tetrahydromethanopterin $\mathrm{N}$-formyltransferase, Formate dehydrogenase, Anaerobic carbon monoxide dehydrogenase and Catalase-peroxidase were associated with methane metabolism. K00600 module and Enzyme Commissions (1.11.1.6 \& 2.1.2.1) were common for dung and rumen fluid's enzymatic pathways. Functional analysis for the enzymes identified were significant $(P<0.05)$ for 5 metabolism processes. Breeding for tolerable methane emitting dairy cattle for a sustainable environment should be undertaken.

\section{Introduction}

The livestock industry contributes to the subsistence of more than one billion of the world's poorest people and supports approximately 1.1 billion people (Hurst et al., 2005). Rearing livestock is an effective risk mitigation strategy for vulnerable populations, and an important nutrient and traction source for smallholder farming systems (Thornton, 2010). Despite there being great disparities between developed and developing countries, livestock products make up 33\% of consumed protein and $17 \%$ of consumed kilocalories globally (Rosegrant et al., 2009). It is estimated that, by 2050, milk and beef production will increase by 191 and 153 percent, respectively $(F A O, 2018)$. This will lead to an increased need to improve productivity to cater for the increased food demand. However, producing food intensively is likely to place more burden on the environment, as it may increase the amount of greenhouse gases (GHG) produced by the animals.

Methane $\left(\mathrm{CH}_{4}\right)$ is a greenhouse gas with a 28-times global warming potential than that of carbon dioxide $\left(\mathrm{CO}_{2}\right)$ (IPCC et al., 2014). The main sources of methane emissions from anthropogenic activities are carbon dioxide and ruminants. The livestock sector contributes $14.5 \%$ of global GHG emissions (Gerber et al., 2013). In ruminants, $\mathrm{CH}_{4}$ is produced after reduction of $\mathrm{CO}_{2}$ using $\mathrm{H}_{2}$ by methanogenic archaea (Danielsson et al., 2012). Methanogenic archaea are part of symbiotic microorganisms that aid in the breakdown of consumed complex carbohydrates from plants to simple molecules that can be utilized by the ruminant animals (Danielsson et al., 2012; St- Pierre \& Wright, 2013). The microorganisms in this mutual association are bacteria, protozoa, and fungi. The host facilitates the microorganisms' physical and chemical requirements (Stewart et al., 2018), while the microbes break down complex compounds to 
produce volatile fatty acids (VFA) and varying amounts of formic acid, hydrogen $\left(\mathrm{H}_{2}\right)$ and $\mathrm{CO}_{2}(\mathrm{Hook}$ et al., 2010). Methane production by the methanogenic archaea process holds $\mathrm{H}_{2}$ partial pressure down, leading to lesser fermentation of reduced end products like acetate (Moss et al., 2008). The produced methane is not utilized by the animal itself; rather it is eructed from the animal to the atmosphere. Methane is therefore not only harmful to the environment, it also represents a proportion of 2 to $12 \%$ gross energy loss to the animal (Johnson \& Johnson, 1995).

In recent years, the knowledge on gut microbiology has risen due to new molecular techniques such as next-generation sequencing, but the methanogens from the guts of crossbred dairy animals in developing countries have not been well characterized and their connection to $\mathrm{CH}_{4}$ levels remains largely unaddressed. Moreover, given the differences in the physiological status of various sections of the cattle gut, it is unclear which microbial genes are responsible for methane production in these various sections of the gut of cattle reared by small holder dairy cattle and who are exposed to sharp fluctuations in feed(s). To address these questions, we studied cows reared by small holder dairy farmers in the tropics that had different genotypes and were reared as different herds to identify gut methanogens from the rumen fluid and dung samples and functional enzymes associated with methane production and metabolism.

\section{Materials And Methods}

\subsection{Description of the experimental sites}

The research was undertaken in one experimental site in Kenya (University of Nairobi (UON) Faculty of Veterinary Medicine, Kanyariri farm, Kiambu County) and two sampling sites in Tanzania (Rungwe and Lushoto districts). The Tanzanian sampling sites and herd were under a Tanzanian led Project by International Livestock Research Institute (ILRI) dubbed "Maziwa Zaidi platform".

\subsection{Sample collection from the experimental animals}

Individual fecal samples were collected from 18 crossbred dairy cows from each district in Tanzania and from six animals reared at the UON Veterinary and teaching farm. The Tanzanian's herd was purposefully selected based on the animal's genotypes (Cheruiyot et al., 2018), and willingness of the dairy farmers to participate in the research project. The collection of fecal samples was done by palpating the rectum using clean sterilized lubricated hand sleeves. About $250 \mathrm{~g}$ of fecal matter was hand grabbed and a proportion transferred into a sterile labelled $50 \mathrm{ml}$ falcon tube as described by Kibegwa et al., (2020). About $250 \mathrm{ml}$ of rumen fluid was collected using a flexible stomach tube from each animal of the six experimental animals at UON Veterinary and teaching farm immediately after collection of fecal samples. The first $200 \mathrm{ml}$ of the rumen fluid was then discarded to avoid salivary contamination and the remaining $50 \mathrm{ml}$ transferred into sterile labelled falcon tubes. The samples from each sampling were immediately placed in a cool box with ice cubes and shipped to the Biosciences East and Central Africa (BecA-ILRI) Hub laboratories, Nairobi campus, where they were stored at $-20^{\circ} \mathrm{C}$ until further processing. 


\subsection{DNA Extraction and Illumina sequencing library preparation}

Before DNA extraction, samples were thawed at room temperature and then whirl wound thoroughly at maximum speed for at least 30 seconds as described by Habimana et al., 2018 for homogeneity. Whole genomic DNA was extracted from individual samples using the QIAamp DNA Stool Mini Kit (Qiagen, USA) following the manufacturer's instructions. Nucleic acid quality and quantity was assessed using NanoDrop ${ }^{\circledR}$ ND-2000 UV spectrophotometer (Nano-Drop Technologies, Wilmington, DE) and 1\% Agarose gel electrophoresis. Library construction was performed using the Nextera DNA Preparation Kit and Nextera index Kit (Illumina, San Diego, CA, USA), following the manufacturer's instructions. In summary, the procedure involved two PCR procedures. In PCR one, 50ng of the genomic DNA was initially fragmented and adapters added simultaneously. The second PCR was limited (5 cycles) PCR to amplify the fragments of interest. After the first PCR, the fragments were purified using the Zymo DNA Kit (Zymo Research Corporation, Irvine, CA, USA) while after the second PCR, the product was cleaned up and size selected using AMPure XP beads (A63881, Beckam Coulter, Brea, CA, USA). The final library concentration and the average library size was measured using the Qubit ${ }^{\circledR}$ HS Assay Kit (Life Technologies Corporation, Grand Island, NY, USA), and Bioanalyzer tapestation 2200 (Agilent Technologies, Santa Clara, USA), respectively. Paired end (200 cycles) sequencing of pooled equimolar libraries was done using the Illumina MiSeq v3 (Illumina) System at the Biosciences eastern and central Africa (BecA)-ILRI Hub.

\subsection{Methanogens taxonomic characterization and identification of enzymes and pathways associated with methane metabolism}

All reads were uploaded to the ILRI research computing cluster (https://hpc.ilri.cgiar.org/), were analyzed and taxonomic assignment was done. Prior to taxonomic assignment, evaluation of sequence quality was done using FastQC software v.0.11.5

(https://www.bioinformatics.babraham.ac.uk/projects/fastqc/), poor quality reads were truncated using FASTX-trimmer, within the FASTX-toolkit v.0.0.14 (https://hannonlab.cshl.edu/ fastx.toolkit/). Sequences that had passed quality control were uploaded to MG-RAST (metagenomics Rapid Annotation using Subsystem Technology) for detection of taxa. Quality filtered sequences were then assembled into contigs using SPAdes version 3.13.0. (https://github.com/ablab/spades). SPAdes-assembled contigs were uploaded to Kyoto Encyclopedia of Genes and Genomes (KEGG) reference database (Kenahisa and Goto, 2000). Functional genes were identified from the database with the KEGG Automatic Annotation Server (KAAS) version 2.1 (KAAS query list) as previously shown by Auffret et al. (2017). The contigs from each sample were separately blasted to a dataset of prokaryotes based on the assignment method of a bidirectional best hit and then computed and the structural genes checked via https://www.kegg.jp. The KEGG IDs were picked and imported to the Ipath3 to visualize their involvement in metabolism of 
microbial metabolism in diverse environment(s). The statistical analysis was performed using IBM SPSS statistics 20 at $P<0.05$.

\section{Results}

Generally, the relative total abundance (\%) of the methanogens from dung samples were Kenyan dung (43.56\%), Lushoto (30.78\%), and Rungwe (9.76\%). Taxonomic classes Archaeoglobi, Thermococci, and Thermoprotei showed relative abundance only in the dung and not in the rumen for the case of Kenyan samples (Fig. 1).

The taxonomic class Methanomicrobia was dominant ( 39\%) from the rumen and from the dung samples ( 44\%) (Supplementary File 1 and 2). Taxanomic classes: Methanomicrobia and Methanobacteria had more abundance of the species in the dung when compared to their presence in the rumen. The other taxonomic classes had a higher abundance in the rumen other than in the dung (Supplementary File 3). The 5 phyla identified according to the relative abundance during the study were Euryarchaeota (95.37\%), Crenarchaeota (3.74\%), Thaumarchaeota (0.52\%), Korarchaeota $(0.31 \%)$ and Nanoarchaeota (0.06\%) (Supplementary File 4).

This study identified some species that were candidates for taxonomic classification. A higher relative abundance (\%) of the candidates were from Tanzanian samples (Fig. 2). Some species were noted missing out to the higher taxonomic class but got assigned at the genus level. A proportion of $\sim 6 \%$ of the rumen samples were unclassified, while $\sim 4 \%$ of the dung samples were not classified. At the lower level of classification, averagely $\sim 7.77 \%$ (at the genus level) of the species remained unassigned. Of the unassigned Archaeal methanogens (at the genus level), Kenyan samples had a collective relative abundance of $7.04 \%$ (dung) and $9.94 \%$ (rumen) while Tanzanian had a proportion of $7.7 \%$ (Lushoto) and Rungwe $7.75 \%$ (Fig. 3). Most of the unassigned archaeal species were from the genera Cenarchaeum and Nitrosopumilus. The Class Aciduliprofundum was noted to have candidates for classification. The scholars Duya et al., (2020) and Wemheuer et al., (2019) during their studies identified the taxonomic family Nitrosopumilaceae being dominant, which was different during this study as members of the said family had species who are not fully classified.

Methanogens from 5 phyla (Archaeal domains) related to methane release and metabolism were identified. They were Euryarchaeota, Crenarchaeota, Thaumarcheota, Korarchaeota, and Nanoarchaeota. Crenarchaeota and Euryarchaeota phyla were statistically significant $(P<0.05)$ in the rumen and dung samples. Methanogens from the following 3 genera; Methanocorpusculum, Methanosarcina, and Methanococcus were statistically significant (Table 1). 
Table 1

Analysis for the presence of methanogens from the Rumen Fluid versus dung samples from Kenya

\begin{tabular}{|c|c|c|c|}
\hline Phylum/Genus & Rumen & Dung & P-value \\
\hline Crenarchaeota & $4.6 \pm 0.15$ & $2.67 \pm 0.17$ & $<0.01$ \\
\hline Sulfolobus & $0.58 \pm 0.1$ & $1.05 \pm 0.14$ & 0.03 \\
\hline Pyrobaculum & $0.45 \pm 0.06$ & $0.47 \pm 0.13$ & 0.91 \\
\hline Thermofilum & $0.35 \pm 0.02$ & $0.53 \pm 0.1$ & 0.13 \\
\hline Staphylothermus & $0.14 \pm 0.03$ & $0.28 \pm 0.11$ & 0.26 \\
\hline Caldivirga & $0.15 \pm 0.05$ & $0.17 \pm 0.08$ & 0.85 \\
\hline Euryarchaeota & $94.6 \pm 0.3$ & $96.61 \pm 0.2$ & $<0.01$ \\
\hline Methanobrevibacter & $20.62 \pm 2.31$ & $14.74 \pm 1.34$ & 0.07 \\
\hline Methanocorpusculum & $22.01 \pm 2.51$ & $4 \pm 0.22$ & $<0.01$ \\
\hline Methanosarcina & $10.25 \pm 0.49$ & $14.05 \pm 0.65$ & $<0.01$ \\
\hline Methanococcus & $6.16 \pm 0.11$ & $8.49 \pm 0.71$ & 0.02 \\
\hline Methanothermobacter & $3.39 \pm 0.14$ & $3.07 \pm 0.17$ & 0.19 \\
\hline Thaumarchaeota & $0.45 \pm 0.2$ & $0.52 \pm 0.07$ & 0.75 \\
\hline Cenarchaeum & $0.14 \pm 0.05$ & $0.1 \pm 0.04$ & 0.53 \\
\hline Nitrosopumilus & $0.38 \pm 0.05$ & $0.35 \pm 0.19$ & 0.88 \\
\hline Korarchaeota & $0.31 \pm 0.13$ & $0.15 \pm 0.02$ & 0.3 \\
\hline Nanoarchaeota & $0.04 \pm 0.02$ & $0.04 \pm 0.02$ & 0.97 \\
\hline
\end{tabular}

No variation $(P<0.05)$ in the relative abundance of methanogens in the dung from the study areas was noted (Table 2 and Table 3 ). 
Table 2

Analysis of dung methanogens in Kenya versus Tanzania analysis

\begin{tabular}{|llll|}
\hline Phylum/Genus & Kenya & Tanzania & P-value \\
\hline Crenarchaeota & $2.59 \pm 0.22$ & $4.45 \pm 0.39$ & 0.18 \\
\hline Sulfolobus & $1.05 \pm 0.14$ & $1.44 \pm 0.18$ & 0.49 \\
\hline Pyrobaculum & $0.47 \pm 0.13$ & $0.73 \pm 0.09$ & 0.34 \\
\hline Thermofilum & $0.53 \pm 0.1$ & $0.33 \pm 0.05$ & 0.17 \\
\hline Staphylothermus & $0.28 \pm 0.11$ & $0.28 \pm 0.06$ & 0.99 \\
\hline Caldivirga & $0.17 \pm 0.08$ & $0.26 \pm 0.05$ & 0.54 \\
\hline Euryarchaeota & $96.63 \pm 0.28$ & $94.44 \pm 0.44$ & 0.17 \\
\hline Methanobrevibacter & $14.74 \pm 1.34$ & $25.38 \pm 2.7$ & 0.2 \\
\hline Methanocorpusculum & $4 \pm 0.22$ & $6.39 \pm 0.98$ & 0.42 \\
\hline Methanosarcina & $14.05 \pm 0.65$ & $12.83 \pm 0.82$ & 0.63 \\
\hline Methanococcus & $8.49 \pm 0.71$ & $6.32 \pm 0.39$ & 0.08 \\
\hline Methanothermobacter & $3.07 \pm 0.17$ & $3.85 \pm 0.29$ & 0.38 \\
\hline Thaumarchaeota & $0.57 \pm 0.06$ & $0.54 \pm 0.06$ & 0.88 \\
\hline Cenarchaeum & $0.1 \pm 0.04$ & $0.26 \pm 0.05$ & 0.31 \\
\hline Nitrosopumilus & $0.35 \pm 0.19$ & $0.28 \pm 0.05$ & 0.66 \\
\hline Korarchaeota & $0.16 \pm 0.03$ & $0.49 \pm 0.08$ & 0.23 \\
\hline Nanoarchaeota & $0.04 \pm 0.02$ & $0.08 \pm 0.03$ & 0.72 \\
\hline
\end{tabular}


Table 3

Analysis of the dung methanogens in the Tanzanian samples.

\begin{tabular}{|llll|}
\hline Phylum/Genus & Rungwe & Lushoto & P-value \\
\hline Crenarchaeota & $4.68 \pm 0.63$ & $4.21 \pm 0.48$ & 0.56 \\
\hline Sulfolobus & $1.4 \pm 0.26$ & $1.48 \pm 0.27$ & 0.84 \\
\hline Pyrobaculum & $0.74 \pm 0.1$ & $0.72 \pm 0.14$ & 0.93 \\
\hline Thermofilum & $0.29 \pm 0.07$ & $0.37 \pm 0.06$ & 0.38 \\
\hline Staphylothermus & $0.22 \pm 0.06$ & $0.34 \pm 0.1$ & 0.32 \\
\hline Caldivirga & $0.3 \pm 0.07$ & $0.21 \pm 0.05$ & 0.36 \\
\hline Euryarchaeota & $94.19 \pm 0.66$ & $94.69 \pm 0.61$ & 0.58 \\
\hline Methanobrevibacter & $22.4 \pm 3.43$ & $28.36 \pm 4.16$ & 0.28 \\
\hline Methanocorpusculum & $7.83 \pm 1.73$ & $4.95 \pm 0.81$ & 0.14 \\
\hline Methanosarcina & $13.2 \pm 1.21$ & $12.45 \pm 1.12$ & 0.66 \\
\hline Methanococcus & $6.75 \pm 0.57$ & $5.88 \pm 0.51$ & 0.27 \\
\hline Methanothermobacter & $3.48 \pm 0.41$ & $4.22 \pm 0.39$ & 0.2 \\
\hline Thaumarchaeota & $0.52 \pm 0.08$ & $0.56 \pm 0.1$ & 0.76 \\
\hline Cenarchaeum & $0.28 \pm 0.08$ & $0.24 \pm 0.06$ & 0.73 \\
\hline Nitrosopumilus & $0.28 \pm 0.07$ & $0.28 \pm 0.06$ & 0.96 \\
\hline Korarchaeota & $0.54 \pm 0.12$ & $0.43 \pm 0.1$ & 0.47 \\
\hline Nanoarchaeota & $0.06 \pm 0.03$ & $0.1 \pm 0.06$ & 0.54 \\
\hline Significant (P<0.05) variation among methanogens (16 species) in the rumen and dung was also & \\
\hline noted (Table 4). & & & \\
\hline
\end{tabular}


Table 4

Significantly varying methanogen species between rumen and dung samples

\begin{tabular}{|llll|}
\hline Phylum/Species & Rumen & Dung & P value \\
\hline Euryarchaeota & & & \\
\hline Methanobrevibacter smithii & $8.08 \pm 0.92$ & $12.35 \pm 1.01$ & 0.02 \\
\hline Methanosarcina acetivorans & $5.82 \pm 0.38$ & $4 \pm 0.35$ & 0.01 \\
\hline Methanocorpusculum labreanum & $4 \pm 0.22$ & $22.01 \pm 2.51$ & $<0.01$ \\
\hline Methanosarcina mazei & $3.17 \pm 0.31$ & $2.31 \pm 0.05$ & 0.03 \\
\hline Methanospirillum hungatei & $2.93 \pm 0.14$ & $1.91 \pm 0.2$ & 0.01 \\
\hline Methanococcoides burtonii & $2.93 \pm 0.07$ & $2.04 \pm 0.15$ & $<0.01$ \\
\hline Aciduliprofundum boonei & $2.6 \pm 0.18$ & $0.99 \pm 0.04$ & $<0.01$ \\
\hline Methanococcus vannielii & $2.55 \pm 0.13$ & $1.78 \pm 0.09$ & $<0.01$ \\
\hline Archaeoglobus fulgidus & $1.98 \pm 0.42$ & $0.86 \pm 0.06$ & 0.04 \\
\hline Thermococcus kodakarensis & $1.75 \pm 0.15$ & $0.75 \pm 0.1$ & $<0.01$ \\
\hline Thermoplasma acidophilum & $0.93 \pm 0.19$ & $0.34 \pm 0.05$ & 0.02 \\
\hline Thermoplasma volcanium & $0.83 \pm 0.11$ & $0.43 \pm 0.03$ & 0.01 \\
\hline Haloterrigena turkmenica & $0.8 \pm 0.13$ & $0.35 \pm 0.08$ & 0.03 \\
\hline Methanothermococcus okinawensis & $0.3 \pm 0.04$ & $0.12 \pm 0.03$ & 0.01 \\
\hline Crenarchaeota & & & \\
\hline Hyperthermus butylicus & $0.33 \pm 0.05$ & $0.14 \pm 0.02$ & 0.01 \\
\hline Ignisphaera aggregans & $0.31 \pm 0.03$ & $0.13 \pm 0.01$ & $<0.01$ \\
\hline Shows the metabolic functions & & & \\
\hline
\end{tabular}

Generally, Fig. 4 shows the metabolic functions that were affected in the rumen and dung samples by archaea derived from Ipath3. The pathway contributed by the functionality of the rumen samples was denoted by a green color, that from the dung was red, and those that overlapped was shown by the blue color.

Green represents functionality in the rumen affected by the enzymes generally, Blue represents the overlap of both the rumen and the dung and red represents dung functional enzymes in the metabolic pathways

Fig. 5 shows the enzymes of importance that functioned and/or affected in the samples from the dung within the methane metabolism pathways. The 5 enzyme commissions (EC) were: ECs 1.11.1.6, 2.1.2.1, 1.2.99.2 (Now referred as 1.2.7.4), 2.3.1.101 (Now referred as 1.17.1.9) and 1.2.1.2. The specific enzyme commissions were denoted by red stars on the box ECs. Enzymes with functionality in the rumen during 
methane metabolism were shown in Fig. 6 and the specific functional ECs (1.11.1.6 and 2.1.2.1) are shown by the red stars. The two functional ECs in the rumen and the KO0600 (methane metabolism) module pathway were common for both the rumen and dung samples.

The module KAAS database also showed functionality for metabolism (09100) and energy metabolism (09102). Modules describing methanogenesis using methanol (M00356), acetate (M00357), and methylamine/ dimethyamine (MO0563) or Carbon (IV) Oxide (MO0567) as in the KEGG pathway were noted alongside the stated ECs above. KEGG Orthology (KO) in the rumen were 271 ID entries while the $\mathrm{KO}$ in the dung were $411 \mathrm{IDs}$. The entry IDs had different metabolic functions. On the functional analysis level for 2 selection criteria of MGRAST, Fig. 7 shows 5 statistically significant $(P<0.05)$ metabolisms both for the rumen fluid and dung samples that were generated. They are carbohydrate metabolism, biosynthesis of other secondary metabolites, xenobiotics biodegradation and metabolism, nucleotide metabolism, and amino acid metabolism. Bolded values were statistically significant $(P<0.05)$ for the functional level 2 from the MGRAST analysis

\section{Discussion}

\subsection{Gut Methanogens}

Methanogens are microorganisms initially exclusively thought to be from the phylum Euryarchaeota. From the recent documentation, there has been advancement to other different phyla. Some of the phyla already agreed upon are Thaumarchaeota, Aigarchaeota, Crenarchaeota, Korarchaeota (TACK), Nonarchaeota, Bathyarchaeota, Geoarchaeota, Marsarchaeota, and Verstraetearchaeota (Berghuis et al., 2019; Vanwonterghem et al., 2016; Evans et al., 2015; Kelly et al., 2011). This study identified species from TACK, Euryarchaeota, and Nonarchaeota. Phyla Euryarchaeota and Crenarchaeota were noted to be dominant among archaeal methanogens and a few presences of methanogens from the phyla Thaumarchaeota, Korarchaeota and Nanoarchaeota. The same methanogen abundance was noted by Jia et al., (2017). Jia et al., (2017) further noted that the phylum Euryarchaeota (specifically those from the genus Methanosarcina) was notably responsible for a variety of functionalities related to methane biosynthesis. The genus Methanosarcina possibly will produce methane with the help of enzymes and protein constituents in the methyl nutrient pathway, acetic acid, and $\mathrm{CO}_{2}$ reduction (Thauer, 2011). Methanogens occupy various diverse environments ranging from hostile environments to favorable conditions. They are acidophilic mesophiles and/or psychrophiles (Evans et al., 2015). Their vast environmental exposure would cause them to utilize different types of metabolism for their nourishment. Some of the phyla were not identified during this study because of the high temperature requirement that the gut of the bovines do not offer as their optimal conditions are between $38.8^{\circ} \mathrm{C} \pm 0.5^{\circ} \mathrm{C}$ (Chen et al., 2018) and/or their full information and categorization has not been fully documented. The Phylum Bathyarchaeota, for example, which was initially identified as Miscellaneous Crenarchaeotal group, has a small proportion of its species cultured and their genomic information completed, calling for advance work (Dayu et al., 2020; Meng et al., 2014; Lloyd et al., 2013). 
Metharchaeal methanogens have been exclusive studied in comparison to other phyla. They have been recorded to have 155-200 isolated species which are clustered into 4 classes, 7 orders, 14 families, and 29-35 diverse genera (Singh et al., 2011). This study showed presence for 3 taxonomic classes, 6 orders, 12 families, 24 genera and 37 individual species of the Metharchaeal methanogens. This is an illustration of a high representation of the metharchaeal methanogens in the study areas. Methanogens in the rumen and rectal area of the ruminant species vary in their population's organization, ecology, and their substrate sources (Knapp et al., 2014). Such variation could be because of the prevailing physicochemical properties (Dayu et al., 2020). The most dominant species in the fore and hindgut was noted to be Methanocorpusculum labreanum ( 17\%), followed by other hydrogenotrophic methanogens which is in agreement with a study by Auffret et al., (2017) and Chen et al., (2014). The dominance of Methanocorpusculum can be explained by their ability to utilize a wide range of substrates such as acetate, $\mathrm{H}_{2}+\mathrm{CO}_{2}$, formate, ethanol, 2-propanol, 2- butanol, or cyclo-pentanol. Methanocorpusculum relies on acetate as a growth feature and on peptone, tungstate, and nickel for their stimulatory (Rosenberg et al., 2014). Moreover, this genus can survive in a wide environment, temperature range of $15-60^{\circ} \mathrm{C}$ and $\mathrm{pH}$ of 6.1-8.0 (Liu and Whitman, 2008), all of which can be achieved in the rumen.

Methanococcus are methanogens that were thought to be only isolated from the sea. However, that opinion since changed when they were found in other environments that are not like marine conditions (Tumbula and Whitman, 2003). These species are not associated with any disease on their hosts and are firmly anaerobic and hydrogenotrophic. They have distinct abilities to undertake sulfur metabolism (Liu et al., 2009). Most are mesophilic (require a temperature of between $20-45^{\circ} \mathrm{C}$ ), and others are thermophilic $\left(41-122^{\circ} \mathrm{C}\right)$ or hyperthermophilic (above $\left.60^{\circ} \mathrm{C}\right)($ Stetter, 2006). In this study, mesophilic species:

Methanococcus aeolicus, Methanococcus maripaludis, Methanococcus vannielii, and Methanococcus voltae were identified. The species identified in this study were similar to those identified by Goyal et al., (2016).

Methanosarcina metarchaeals utilize the substrates acetate, $\mathrm{H}_{2}+, \mathrm{CO}_{2}, \mathrm{CO}$, methanol, methylamines, and metylmercaptoproprionate dismetylsulfide. They grow on a variety of substrates because they are notably cytochrome bound (Buan et al., 2011). The methanogens here operate in a temperature range of between $1^{\circ} \mathrm{C}-70^{\circ} \mathrm{C}$ and a $\mathrm{pH}$ range of $4-10$ and adapt to low hydrogen availability (Liu and Whitman, 2008). Methanosarcinas have a flexible metabolic pathway making their growth genes on one substrate easily deleted without affecting their subsequent growth on another available substrate(s). With this capability, methanosarcina's genetic analysis can be used to investigate how methanogens grow and participate in methane production along the known methane metabolism pathways (Kulkarni et al., 2009). Methanosarcina acetivorans, Methanosarcina barkeri, and Methanosarcina mazei were identified during this study.

The methanogen Methanocorpusculum labreanum species that hailed from Methanocorpusculum was identified during this study. This species was found both among the rumen fluid samples and dung samples from the Kenyan dairy cattle. This was in contrast with a study by Liu et al., (2012) in a study on Chinese sheep that identified sequences related to Methanocorpusculum species from sheep droppings 
only but absent in the rumen of the same sheep. In another study by Luo et. al., (2013), the authors found out that Methanocorpusculum were dominant $(60 \%)$ in the hindgut of captive Ceratotheriumsimum. Methanocorpusculum utilizes the substrate acetate, $\mathrm{H}_{2}+\mathrm{CO}_{2}$, formate, ethanol, 2-propanol, 2- butanol, or cyclo-pentanol. Species from this genus were also identified from a wastewater bio digester (Oren, 2014). Identification of this species in the rumen indicated that they may have a wider niche than previously thought.

\subsection{Functional enzymes associated with methane metabolism}

Enzymes catalyze chemical reactions that are key for life functionality such as metabolism and digestion (Blanco and Blanco, 2017). Over the years, there is an advancement of knowledge on methane metabolism between methanogens and methanotrophic archaea with a universal display of the Methylcoenzyme $M$ reductase complex as a main enzyme in their pathways (Evans et al., 2019). The study identified the function of the enzyme Glycine/Serine hydroxy methyltransferase in the aspects of Amino acid transportation and metabolism/biosynthesis from module entry K00600. This was identified from the Enzyme commission (EC) EC: 2.1.2.1 and was associated with the structural gene glyA. Glycine/Serine hydroxy methyltransferaseis a vitamin reliant enzyme that catalyzes the reversible and conversion of $L$ serine to glycine and tetrahydrofolate to 5, 10-Methylenetetrahydrofolate. Upon completion of the enzymatic reaction, it leads to the delivery of substantial carbon units to the cell (Edgar, 2005). Other studies have noted that this enzyme as well catalyzes glycine and acetaldehyde to form Lthreonine with 4-trimethylammoniobutanal to form 3-hydroxy $\mathrm{N}^{6}, \mathrm{~N}^{6}, \mathrm{~N}^{6}$-trimethyl-L-lysine (Schweitzer et al., 2009). Methanogens like Methanococcus jannaschii, which was identified in this study, have been shown to use the enzyme for amino acid biosynthesis (Tsoka et al., 2004).

Formylmethanofuran-tetrahydromethanopterin- $\mathrm{N}$-formyl transferase enzyme was noted from the EC: 1.17.1.9 (Formerly EC. 2.3.1.101). The module entry involved was K00672. The enzyme was notably involved in energy production and conversion. Entry K00123 that is involved in the anaerobic selenocysteine-containing dehydrogenase was also noted and needed for energy production and conversion. The gene responsible for the enzyme is Ftr. Structural genes associated with the functionality of the enzyme were $f d o G, f d h F$, and $f d w A$. Formylmethanofuran-tetrahydromethanopterin $\mathrm{N}$ formyltransferase enzyme catalyzes two notable substrates; methanofuran and 5-formyl-5-6-7-8tetrahydromethanopterin (Wagner et al. 2016). Methanofuran is the key for methane formation from $\mathrm{CO}_{2}$ by methanogens. $\mathrm{CO}_{2}$ as a methanogenic substrate is initially reduced and activated to formylmethanofuran (Wagner et al., 2016). Mesophilic methanogen (Methanosarcina barkeri) and thermophilic methanogens (Methanopyrus kandleri), that were also identified in this study, have shown functionality for the enzyme (Enzmann et al., 2018).

Anaerobic carbon monoxide dehydrogenase enzyme facilitates the metabolism of methanogens by the reversible interconversion between carbon monoxide and $\mathrm{CO}_{2}$. The catalyzed reaction is vital for energy conservation and carbon fixation among methanogens, especially during the Wood-Ljungdahl pathway 
(King and Weber, 2007; Borrel et al., 2016). Enzyme anaerobic carbon monoxide dehydrogenase of the catalytic subunit was noted during this study from the EC: 1.2.7.4 pathway, formerly EC. 1.2.99.2. The involved module entry was K00198. Hydroxylamine reductase (hybrid-cluster protein) together with the enzymatic function of anaerobic carbon monoxide dehydrogenase are vital for inorganic ion transport and metabolism energy production and conversion. The key genes involved were $\operatorname{coo} S$ and $\operatorname{acs} A$.

Catalase-peroxidase enzyme is documented to be an inconsequential material in the antioxidant system in methanogens even for those aerotolerant, including species such as Methanosarcina acetivorans (Jennings et al., 2014). The catalase-peroxidase enzyme is a sturdy catalase with $\mathrm{H}_{2} \mathrm{O}_{2}$ as the contributor which releases $\mathrm{O}_{2}$ (Vlasits et al., 2010) and molecules of water in a two-step reaction (Nandi et al., 2019). Methanogens in their environments are exposed to oxygen occasionally in aerobic situations, this would call in for the functionality of antioxidants to facilitate lowering levels of oxygen (Angel et al., 2012). Ma and Lu (2011) pointed out that some methanogens can withstand some levels of oxygen for some hours. The enzyme possessed by methanogens (although they are noted to be limited in the numbers- restricted to Methanosarcina species and Methanobrevibacter species) has been noted to have been acquired through a gene ( $k a t G$ ) transferred laterally (Zamocky et al., 2012a). When catalase peroxidase enzyme has functionality in EC: 1.11.1.6, thereby it acts on hydrogen peroxide as an acceptor and not the functionality under EC. 1.11.1.21 of both catalase and peroxidase. Module entry K03782 was involved. Specifically, the Catalase peroxidase I is involved in catalyzing inorganic ion transport and metabolism. The gene involved is katG.

In conclusion, this study broadens our understanding on dominant methanogen species in Kenya and Tanzanian among smallholder cattle and functional enzymes associated with methane metabolism and production. The methanogen species abundances from these study areas in numbers/kind can be utilized exclusively or jointly as indirect selection criteria for methane mitigation. This calls for interdisciplinary cohesion and collaboration for fruitful achievements. Studies should be carried out to taxonomically categorize species missing out of place. Furthermore, every part of the gut (either fore or the hindgut) was capable of hosting methanogens. Targets for methanogens should entirely be towards the whole gastrointestinal tract. Furthermore, there is a need to target functional genes of the microbes and those of animals to achieve a friendly environment without affecting the animal's functionality. Animals who are less methane emitters should be bred to cut on their methane release from the gut. Further studies should be carried out to target pathways for tolerable methane emitter dairy cattle without hindering other necessary metabolic processes. The fraction of the methanogens that are yet to be fully classified should be carried out and a thorough study of their temperature, substrate(s), and pH should be noted.

\section{Declarations}

\section{Author's contribution}


FMK, RCB and CKG conceived and designed the experiment. FMK conducted the experiment. FMK and DKN analyzed the data. DKN wrote the manuscript. All authors read and approved the manuscript.

\section{Acknowledgement}

The authors acknowledge ILRI, Kenya for the support and the facilitation while undertaking this study. Further, we thank the farmers, UON and support personnel who took part during this research work and its analysis.

\section{Funding}

No form of funding was received by the authors to help them during the preparation of this manuscript. This study benefited in kind from the ILRI led project “Maziwa Zaidi” platform.

\section{Financial and non- financial Interests}

All authors declare no financial and non-financial interests

\section{Statement of Ethical statement and Animal Rights}

This research study was performed under the University of Nairobi (UON), Faculty of Veterinary Medicine Institutional Animal Care and Use Committee (IACUC) accepted procedures. Experimental animals were handled professionally while exercising restraint to reduce on any discomfort and injury.

\section{Conflict of Interest Statement}

The authors of this piece of work declares no conflict of interest.

\section{Data availability Statements}

All the data that were generated and/ or analysed during the study are included in the article that has been published and those that are contained in the supplementary files.

\section{References}

1. Angel R, Claus P, Conrad R (2012) Methanogenic archaea are globally ubiquitous in aerated soils and become active under wet anoxic conditions. ISMEJ.; 6: 847-862.

2. Auffret MD, Dewhurst RJ, Duthie CA, Rooke JA (2017) The rumen microbiome as a reservoir of antimicrobial resistance and pathogenicity genes is directly affected by diet in beef cattle

3. Berghuis BA, Yu F, Brian S, Frederik B, Paul C, Woyke T, Quake SR (2019) Hydrogenotrophic methanogenesis in archaeal phylum Verstraetearchaeota reveals the shared ancestry of all methanogens. United States. doi:10.1073/pnas.1815631116

4. Blanco A, Blanco G (2017) Enzymes. Medical Biochemistry 
5. Borrel G, Adam PS, Gribaldo S (2016) Methanogenesis and the Wood-Ljungdahl Pathway: An Ancient, Versatile, and Fragile Association. Genome Biol Evol 8(6):1706-1711. https://doi.org/10.1093/gbe/evw114

6. Buan N, Kulkarni G, Metcalf W (2011) Genetic Methods for Methanosarcina Species. https://doi.org/10.1016/B978-0-12-385112-3.00002-0

7. Chen L, Brugger K, Skovgaard M, Redder P, She Q, Torarinsson E, Greve B, Awayez M, Zibat A, Klenk HP, Garrett RA (2005) The Genome of Sulfolobus acidocaldarius, a Model Organism of the Crenarchaeota. vol 187,14,4992--4999,10.1128/JB.187.14.4992-4999.2005, American Society for Microbiology Journals

8. Chen R, Wang Y, Wei S, Wang W, Lin X (2014) Windrow composting mitigated $\mathrm{CH}_{4}$ emissions: characterization of methanogenic and methanotrophic communities in manure management. Federation of European Microbiological Societies. 90(3):575-586

9. Chen S, Wang J, Peng D et al (2018) Exposure to heat-stress environment affects the physiology, circulation levels of cytokines, and microbiome in dairy cows. Sci Rep 8:14606. https://doi.org/10.1038/s41598-018-328861

10. Cheruiyot EK, Bett RC, Amimo JO, Mujibi DF (2018) Use of a High-Density SNP Chip to Characterize Breed Composition of Crossbred Dairy Cattle in Tanzania Use of a High-Density SNP Chip to Characterize Breed Composition of Crossbred Dairy Cattle in Tanzania. Proceedings of the World Congress on Genetics Applied to Livestock Production;2 (570)

11. Danielsson R, Schnurer A, Arthurson V, Bertilsson J (2012) Methanogenicpopulation and $\mathrm{CH}_{4}$ production in Swedish dairy cows fed different levels of forage. Appl Environ Microbiol 78(17):61726179

12. Dayu Z, Jie P, Zongbao L, Chuanlun Z, Hongbin L, Meng L (2020) The Distribution of Bathyarchaeota in Surface Sediments of the Pearl River Estuary Along Salinity Gradient. Front Microbiol. https://www.frontiersin.org/article/ 10.3389/fmicb.2020.00285

13. Edgar JA (2005) Mice have a transcribed L-threonine aldolase/GLY1 gene, but the human GLY1 gene is a non-processed pseudogene.BMC Genomics

14. Enzmann F, Mayer F, Rother M, Holtmann D (2018) Methanogens: biochemical background and biotechnological applications. AMB Express 8(1):1. https://doi.org/10.1186/s13568-017-0531-x

15. Evans NP, Parks HD, Chadwick GL, Robbins SJ, Orphan VJ, Golding SD (2015) Methane metabolism in the archaeal phylum Bathyarchaeota revealed by genome-centric metagenomics.. Vol. 350:, Issue 434-438.. DOI: 10.1126/science.aac7745. 6259, pp.

16. Evans PN, Boyd JA, Leu AO, etal (2019) An evolving view of methane metabolism in the Archaea. NatRev Microbiol ; 7:219-232

17. FAO (2018) Ethiopia: report on feed inventory and feed balance. FAO, Food and Agriculture Organization of the United Nations, Rome, Italy 
18. Gerber PJ, Henderson B, Makkar H, Hristov A, Oh J, Lee et al (2013) Mitigation of greenhouse gas emissions in livestock production-a review of technical options for non- $\mathrm{CO}_{2}$ emissions

19. Goyal N, Zhou Z, Karimi IA (2016) Metabolic processes of Methanococcus maripaludis and potential applications. Microb Cell Fact 15:107. https://doi.org/10.1186/s12934-016-0500-0

20. Habimana V, Bett RC, Amimo JO et al (2018) Metagenomic analysis of enteric bacterial pathogens affecting the performance of dairy cows in smallholder productions systems. African Journal of Microbiology Research;; 12(17), 387-398.

21. Hook SE, Wright AG, McBride BW (2010) Methanogens: Methane Producers of the Rumen and Mitigation Strategies. https://doi.org/10.1155/2010/945785

22. Hurst $P$, Termine $P$, Karl $M(2005)$ Agricultural workers and their contribution to sustainable agriculture and rural development. FAO, Rome

23. IPCC, 2014: Climate Change 2014: Synthesis Report. Contribution of Working Groups I, II and III to the Fifth Assessment Report of the Intergovernmental Panel on Climate Change [Core Writing Team, Pachauri RK, Meyer LA (eds.)].IPCC, Geneva, Switzerland,151 pp

24. Jennings ME, Schaff CW, Horne AJ, Lessner FH, Lessner DJ (2014) Expression of a bacterial catalase in a strictly anaerobic methanogen significantly increases tolerance to hydrogen peroxide but not oxygen. Microbiology 160(Pt 2):270-278. https://doi.org/10.1099/mic.0.070763-0

25. Jia X, Xi B-D, Li M-X, Yang Y, Wang Y (2017) Metaproteomics analysis of the functional insights into microbial communities of combined hydrogen and methane production by anaerobic fermentation from reed straw. PLoS ONE 12(8):e0183158. https://doi.org/10.1371/journal

26. Johnson KA, Johnson DE (1995) Methane emissions from cattle. J Anim Sci 73:2483-2492. https://doi.org/10.2527/1995.7382483x

27. KAAS query list. www.genome.jp/kaas-bin/kaas. Accessed on 19/6/2019

28. Kanehisa M, Goto S (2000) KEGG: Kyoto encyclopedia of genes and genomes. Nucleic Acids Res 28:27-30

29. Kelly S, Wickstead B, Gull K (2011) Archaeal phylogenomics provides evidence in support of a methanogenic origin of the Archaea and a thaumarchaeal origin for the eukaryotes. Proc. Biol. Sci. 278, 1009-1018. doi:10.1098/rspb.2010.1427 pmid:20880885

30. Kibegwa FM, Bett RC, Gachuiri CK, Stomeo F, Mujibi FD (2020) A Comparison of Two DNA Metagenomic Bioinformatic Pipelines While Evaluating the Microbial Diversity in Feces of Tanzanian Small Holder Dairy Cattle.. doi: 10.1155/2020/2348560. PMID: 32382536; PMCID: PMC7195676.

31. King GM, Weber CF (2007) Distribution, diversity, and ecology of aerobic CO-oxidizing bacteria. Nat Rev Microbiol 5: 107-118. Nature reviews. Microbiology. 5. 107-18. 10.1038/nrmicro1595

32. Knapp JR, Laur GL, Vadas PA, Weiss WP, Tricarico JM (2014) Invited review: Enteric methane in dairy cattle production: Quantifying the opportunities and impact of reducing emissions. J Dairy Sci $97 .$. 3231-3261. Doi 10.3168/jds.2013-7234 
33. Kulkarni G, Kridelbaugh DM, Guss AM, Metcalf WW (2009) Hydrogen is a preferred intermediate in the energy-conserving electron transport chain of Methanosarcina barkeri. Proceedings of the National Academy of Sciences, 106 (37) 15915-15920; DOI: 10.1073/pnas.0905914106

34. Liu C, Zhu ZP, Liu YF, Guo TJ, Dong HM (2012) Diversity and abundance of the rumen and fecal methanogens in Altay sheep native to Xinjiang and the influence of diversity on methane emissions. Arch Microbiol 194:353-361

35. Liu Y, Whitman W (2008) Metabolic, phylogenetic, and ecological diversity of the methanogenic Archaea. Ann NY Acad Sci 1125:171-189.. Annals of the New York Academy of Sciences. 1125. 171-89. 10.1196/annals.1419.019

36. Liu Y, Guo L, Guo R, Wong RL, Hernandez H, Hu J, Chu Y, Amster IJ, Huang L (2009) The Sac10b Homolog in Methanococcus maripaludis Binds DNA at Specific Sites. J Bacteriol 191:2315-2329

37. Lloyd KG, Petersen SchreiberL, Kjeldsen DG, Lever KU, Steen MA, Stepanauskas AD, Richter R, Kleindienst M, Lenk S, Schramm S, Jørgensen A B. B (2013) Predominant archaea in marine sediments degrade detrital proteins. Nature 496:215-218. 10.1038/nature12033 pmid:23535597

38. Luo Y-h, Wright A-DG, Li Y-I, Li H, Yang Q-h, Luo L-j, Yang M-x (2013) Diversity of methanogens in the hindgut of captive white rhinoceroses, Ceratotheriumsimum. BMC Microbiol 13:207

39. Ma K, Lu Y (2011) Regulation of microbial methane production and oxidation by intermittent drainage in rice field soil. FEMS microbiology ecology. 75. 446-56. 10.1111/j.1574-6941.2010.01018. $x$

40. Meng J, Xu J, Qin D (2014) Genetic and functional properties of uncultivated MCG archaea assessed by metagenome and gene expression analyses. ISME J 8:650-659. https://doi.org/10.1038/ismej.2013.174

41. Moss RH, Nakicenovic N, O'Neill BC (2008) Towards New Scenarios for Analysis of Emissions, Climate Change, Impacts, and Response Strategies. Geneva: IPCC. ISBN 978-92-9169-125-8

42. Nandi A, Yan LJ, Jana CK, Das N (2019) Role of Catalase in Oxidative Stress- and Age-Associated Degenerative Diseases. Oxid Med Cell Longev 112019:9613090. doi: 10.1155/2019/9613090

43. Oren A (2014) The Family Methanocorpusculaceae.. In: In: Rosenberg E, DeLong EF, Lory S, Stackebrandt E, Thompson F (eds) The Prokaryotes. Springer, Berlin, Heidelberg. https://doi.org/10.1007/978-3-642-38954-2_314

44. Quehenberger J, Shen L, Albers SV, Siebers B, Spadiut O (2017) Sulfolobus - A Potential Key Organism in Future Biotechnology. Front Microbiol 8:2474. https://doi.org/10.3389/fmicb.2017.02474

45. Rosegrant MR, Ringler C, Sulser TB, Ewing M, Palazzo A, Zhu T, Nelson GC, Koo J, Robertson R, Msangi S, Batka M (2009) Agriculture and Food Security under Global Change: Prospects for 2025/2050. Background note for supporting the development of CGIAR Strategy and Results Framework. International Food Policy Res, Institute: W ashington, D.C

46. Rosenberg E, DeLong EF, Lory S et al (2014) The Prokaryotes. Actinobacteria. Springer-Verlag Berlin, Heidelberg 
47. Schweitzer J, Diesveld MS, Ramon, Etterich H, Eggeling L (2009) The serine hydroxymethyltransferase gene glyA in Corynebacterium glutamicum is controlled by GlyR. J Biotechnol. https://doi.org/10.1016/j.jbiotec.2008.12.008

48. Singh KM, Pandya PR, Parnerkar S (2011) etal.,. Molecular identification of methanogenic archaea from surti buffaloes (Bubalus bubalis), reveals more hydrogenotrophic methanogens phylotypes. Brazilian JournalofMicrobiology.42(1):132-139

49. Stetter K (2006) History of discovery of the first hyperthermophiles. Extremophiles 10:357-362. doi:10.1007/s00792-006-0012-7

50. Stewart RD, Auffret MD, Warr A, Wiser AH, Press MO, Langford KW (2018) etal.,. Assembly of 913 microbial genomes from metagenomic sequencing of the cow rumen. NatureCommunications.; $9(870)$

51. St-Pierre B, Wright ADG (2013) Diversity of gut methanogens in herbivorous animals. Animal 7 Suppl ((1):49-56

52. Thauer RK (2011) Anaerobic oxidation of methane with sulfate: On the reversibility of the reactions that are catalyzed by enzymes also involved in methanogenesis from CO2. Curr Opin Microbiol 14:292-299. 10.1016/j.mib.2011.03.003 pmid:21489863

53. Thornton PK (2010) Review Livestock Production: Recent Trends, Future Prospects. Philosophical Transactions of the Royal Society B: Biological Sciences 365:2853-2867

54. http://dx.doi.org/10.1098/rstb.2010.0134

55. Tsoka S, Simon D, Ouzounis CA (2004) Automated metabolic reconstruction for Methanococcus jannaschii. Archaea (Vancouver, B C) 1(4):223-229. https://doi.org/10.1155/2004/324925

56. Tumbula LD, Whitman BW (2003) Genetics of Methanococcus: possibilities for functional genomics in Archaea. https://doi.org/10.1046/j.1365-2958.1999.01463.x

57. Vanwonterghem I, Evans PN, Parks DH, Jensen PD, Woodcroft BJ, Hugenholtz P, Tyson GW (2016) Methylotrophic methanogenesis discovered in the archaeal phylum Verstraetearchaeota. Nature Microbiology 1(12):1-9. https://doi.org/10.1038/nmicrobiol.2016.170

58. Vlasits J, Jakopitsch C, Bernroitne M, Zamocky M, Furtmüller PG, Obinger C (2010) Mechanisms of catalase activity of heme peroxidases. Archives of Biochemistry and Biophysics. 500 (1): 74-81. doi:10.1016/j.abb.2010.04.018

59. Wagner T, Ermler $\mathrm{U}$, Shima $\mathrm{S}$ (2016) The methanogenic $\mathrm{CO}_{2}$ reducingandfixing enzyme is bifunctional and contains 46 [4Fe-4S] clusters. Science 354:114-117

60. Wemheuer F, von Hoyningen-Huene A, Pohlner M, Degenhardt J, Engelen B, Daniel R, Wemheuer B (2019) Primary Production in the Water Column as Major Structuring Element of the Biogeographical Distribution and Function of Archaea in Deep-Sea Sediments of the Central Pacific Ocean. https://doi.org/10.1155/2019/3717239

61. Zamocky M, Gasselhuber B, Furtmüller PG, Obinger C (2012a) Molecular evolution of hydrogen peroxide degrading enzymes. Arch Biochem Biophys 525:131-144 
Figures

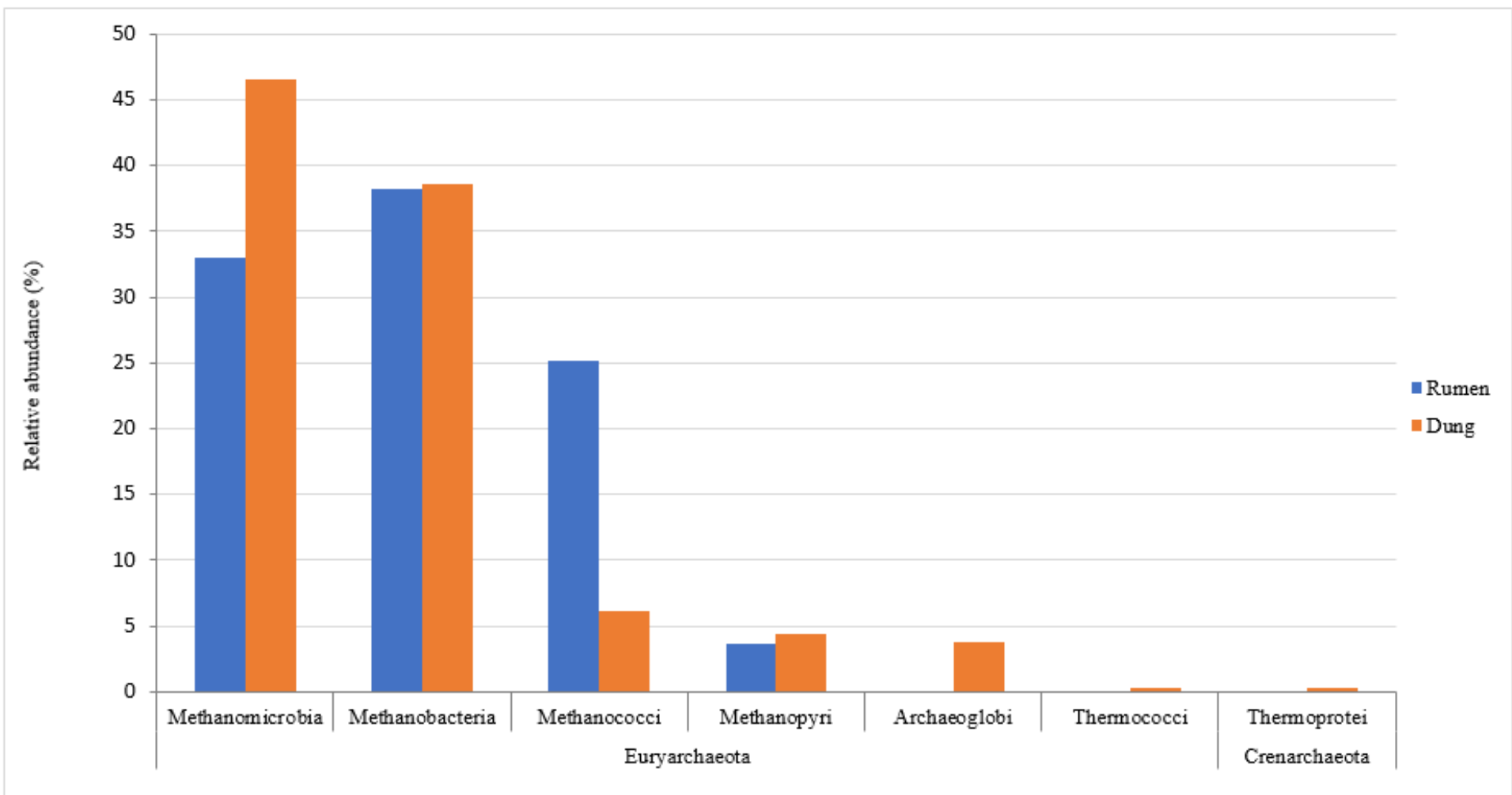

\section{Figure 1}

Relative abundance (\%) of the Euryarchaeota and Crenarchaeota group from the rumen and the dung samples 


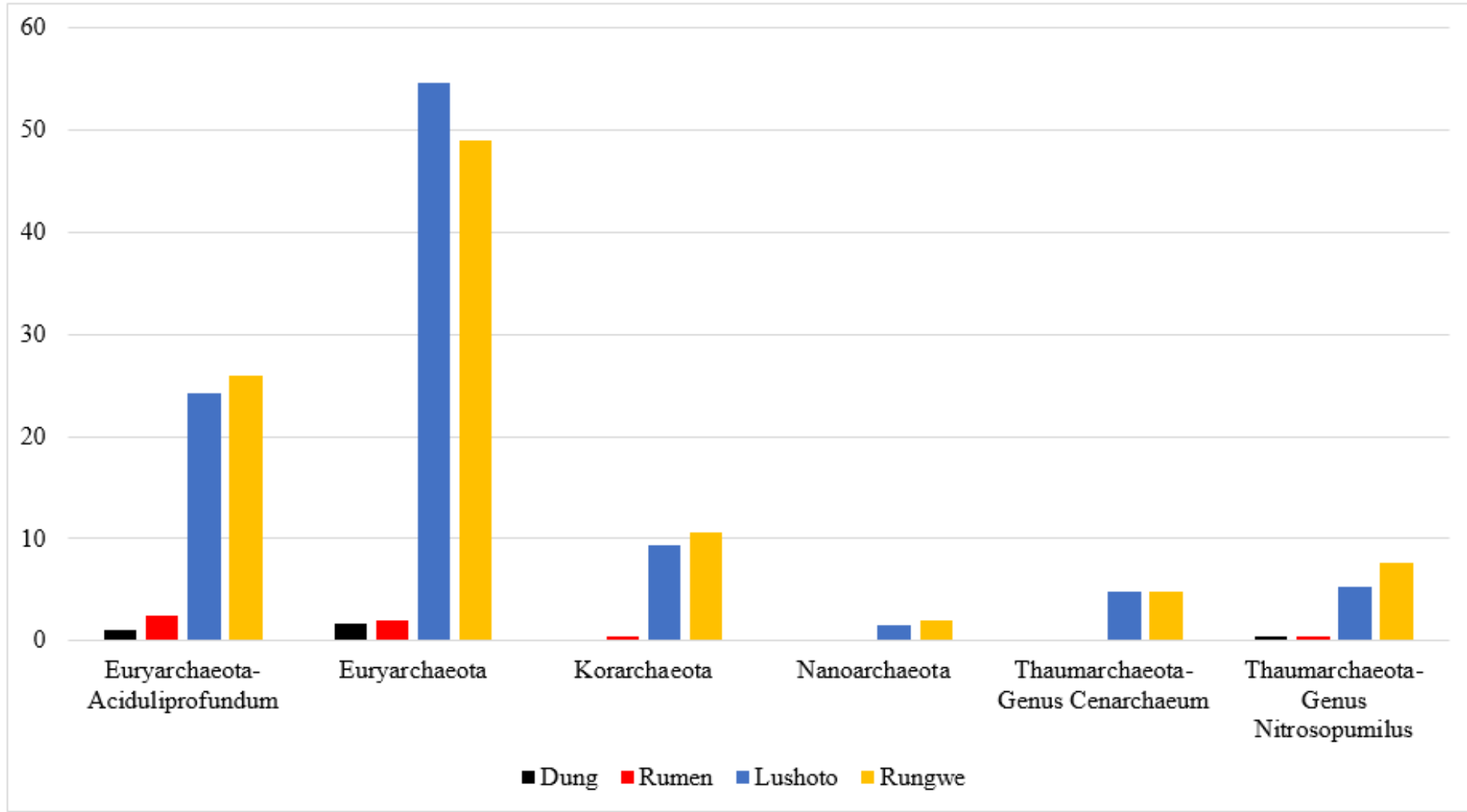

Figure 2

Relative abundance (\%) of the unclassified methanogens from different phyla

\section{Figure 3}

Relative abundance (\%) of the taxonomic classified and unclassified methanogens at the Genus level 


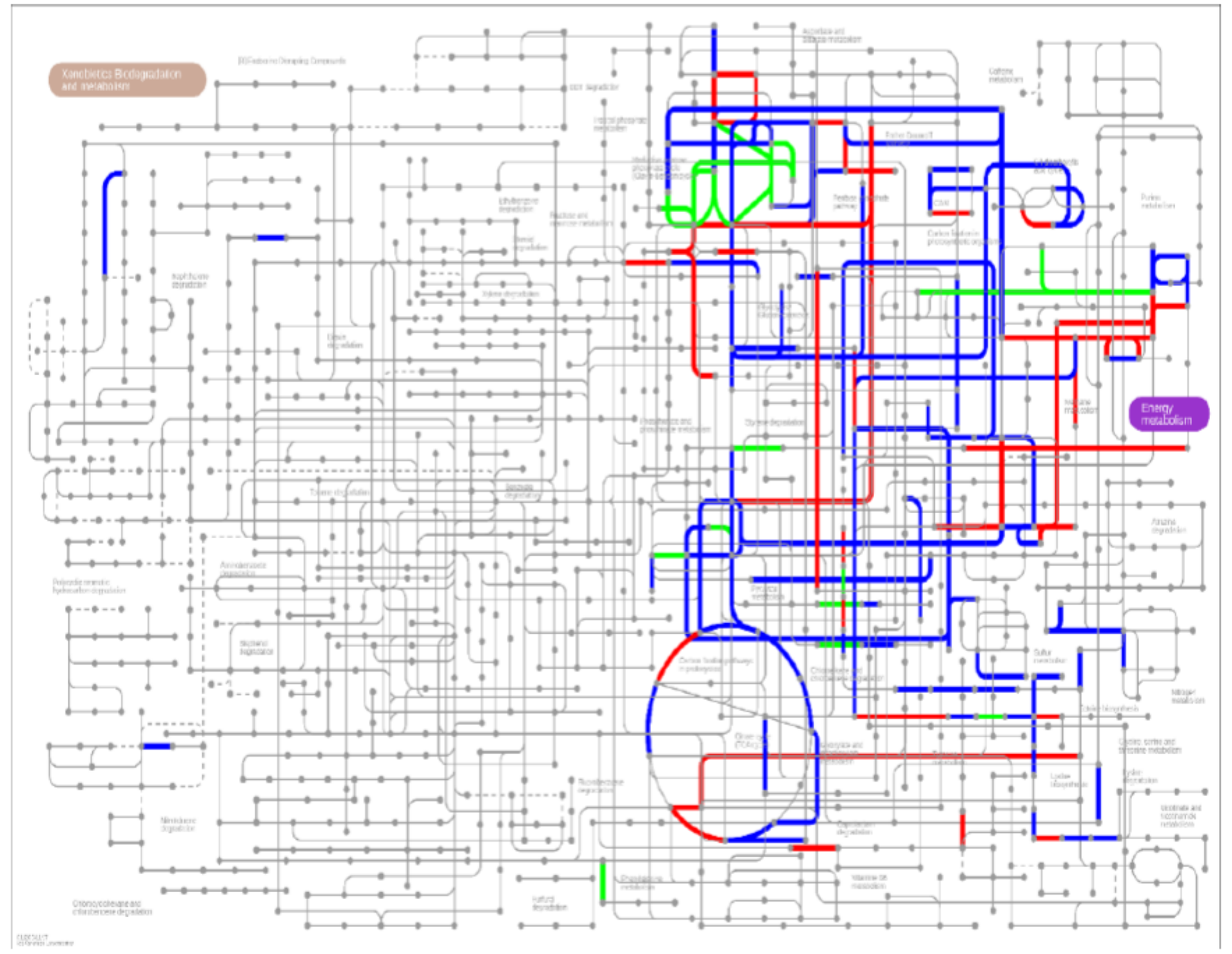

Figure 4

Ipath3 visualization of all metabolic functions affected by Archaea in rumen and fecal samples. 
METHANE METABOLISM

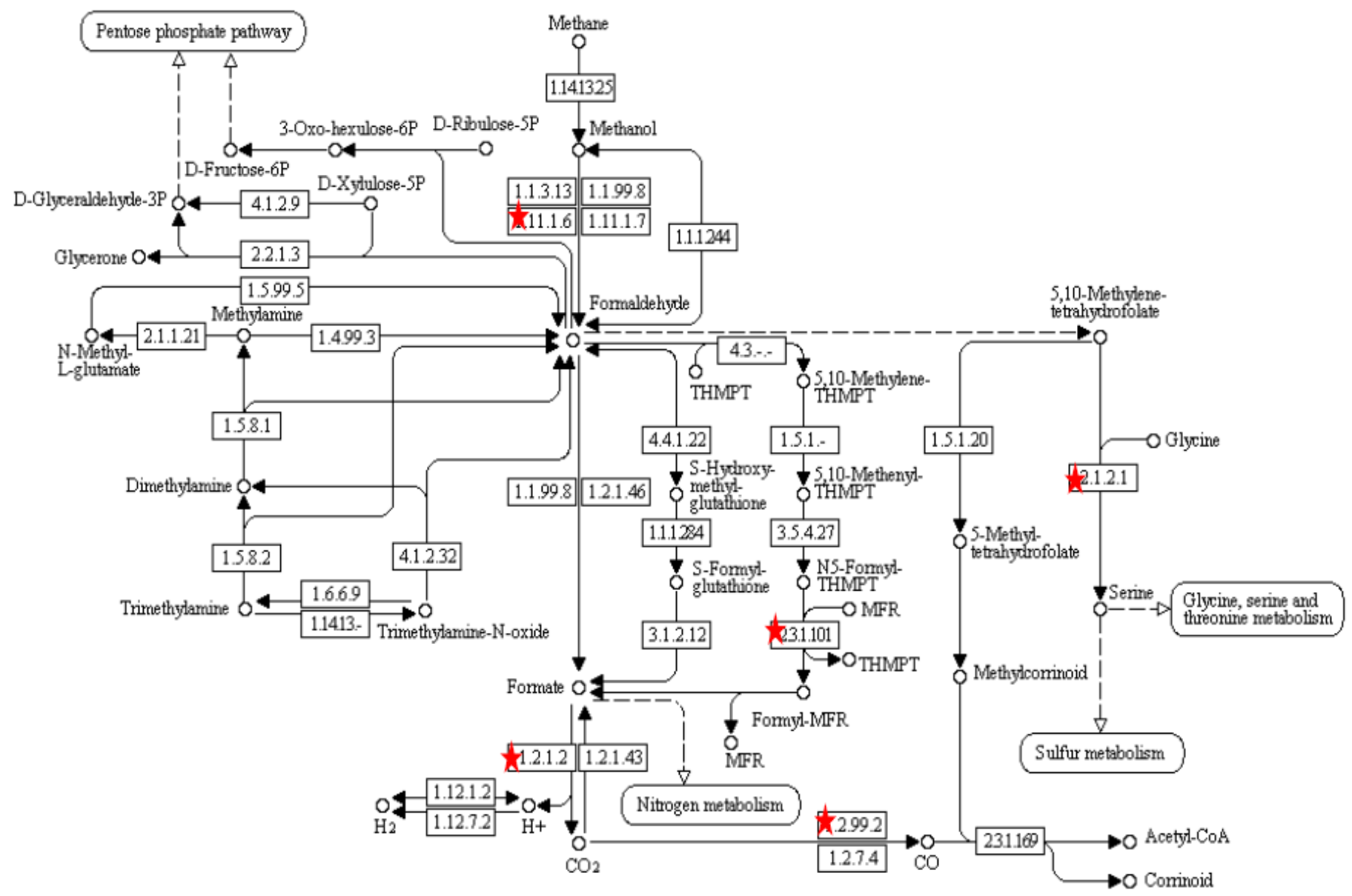

$006809 / 1 / 09$

(c) Kanehisa Laboratories

Figure 5

Enzymes affected in the dung samples within the methane metabolism pathway

Shows the Enzyme commissions affected in the pathway chart

\section{Figure 6}

Enzymes affected in the rumen fluid within the methane metabolism pathway

Shows the enzyme commissions affected during methane metabolism in the chart 
Figure 7

Level 2 of KEGG Orthology (KO) functional analysis between rumen and fecal samples

\section{Supplementary Files}

This is a list of supplementary files associated with this preprint. Click to download.

- Ngetichetal.SupplementaryFiles2021.docx 